\title{
Inseguridad alimentaria en hogares donde habitan adultos mayores. Medellín, Colombia*
}

\author{
Food Insecurity in Households Inhabited by Older Adults, Medellín, Colombia
}

\author{
Insegurança alimentar em lares onde habitam idosos: Medellin, Colômbia
}

Alejandro Estrada-Restrepo'; Nubia Amparo Giraldo-Giraldo²; Gloria Cecilia Deossa-Restrepo³.

1 Magíster en Epidemiología. Universidad de Antioquia. Medellín, Colombia. alejandro.estrada@udea.edu.co. oRciD: https://orcid. org/0000-0002-3261-1282

2 Magíster en Epidemiología. Universidad de Antioquia, Medellín, Colombia. nubia.giraldo@udea.edu.co. oRcil: https://orcid.org/00000001-7795-9947

3 Magíster en Ciencias de la Alimentación y Nutrición Humana. Universidad de Antioquia. Medellín, Colombia. gloria.deossa@udea. edu.co. ORCID: https://orcid.org/0000-0002-1635-1601.

Recibido: 05/08/2020. Aprobado: 06/09/2021. Publicado:20/12/2021

Estrada-Restrepo A, Giraldo-Giraldo NA, Deossa-Restrepo GC. Inseguridad alimentaria en hogares donde habitan adultos mayores. Medellín, Colombia. Rev. Fac. Nac. Salud Pública. 2022;40(1):e342583. Dor: https://doi.org/10.17533/udea.rfnsp. e342583

\section{Resumen}

Objetivo: Determinar la inseguridad alimentaria y algunas características sociodemográficas asociadas a ella, en hogares de Medellín (Colombia) en los que habitaban adultos de 60 años o más. Metodología: Estudio transversal, de muestreo aleatorio estratificado, por nivel socioeconómico y localización geográfica (urbano-rural). Este estudio se basó en 935 hogares, en los cuales habitaba por lo menos un adulto mayor. La seguridad alimentaria se midió con la Escala Latinoamericana y Caribeña de Seguridad Alimentaria. Se utilizaron la prueba chi cuadrado $\left(\chi^{2}\right)$ y odds ratio (OR), con intervalos de confianza, para determinar la asociación de la seguridad alimentaria con las demás variables; además, se elaboró un árbol de clasificación, con el propósito de determinar la importancia relativa de las variables para explicar la seguridad alimentaria. Resultados: El 19,2 \% de los hogares estaba conformado únicamente por adultos mayores. El $63 \%$ de los hogares evaluados presentaron jefaturas femeninas. La inseguridad alimentaria fue del $55 \%$, con mayores proporciones en zonas rurales, estratos bajos y cuando se presenta mayor número de personas por hogar $(p<0,05)$;

Estudio llevado a cabo en el marco del proyecto "Propuesta técnica para el desarrollo del perfil de seguridad alimentaria y nutricional 2015 y la asistencia técnica para la formulación del plan municipal de seguridad alimentaria y nutricional 2016-2028", realizado por Alcaldía de Medellín - Universidad de Antioquia. Fecha de inicio: febrero de 2015; fecha de terminación: diciembre de 2015. 
la inseguridad alimentaria fue más baja en hogares integrados solo por adultos mayores $(\mathrm{OR}=0,58$; $p<0,001)$, con jefes de hogar de 60 años y más $(\mathrm{OR}=0,64 ; p=0,003)$ y hogares que no contaban con programas de ayuda alimentaria dirigidos a adultos mayores $(\mathrm{OR}=0,33 ; p<0,001)$. Conclusión: Este estudio evidencia una alta frecuencia de inseguridad alimentaria en hogares donde viven adultos mayores; además, la edad, el sexo del jefe del hogar y el que este esté conformado solo por adultos mayores son aspectos determinantes de la inseguridad alimentaria. --Palabras clave: adulto mayor, factores sociodemográficos, inseguridad alimentaria, nutrición, salud pública, Medellín (Colombia).

\section{Abstract}

Objective: To determine food insecurity and certain associated sociodemographic characteristics in households inhabited by adults aged 60 years or older in Medellín (Colombia). Methodology: Cross-sectional study using stratified random sampling according to socioeconomic status and geographic area (urban-rural). This study examined 935 households that had at least one older adult. Food security was measured with the Latin American and Caribbean Food Security Scale. The chi-square test $(\chi 2)$ and odds ratio (OR) with confidence intervals were used to determine the relationship between food security and the other variables; additionally, a classification tree was created to identify the relative importance of the variables in explaining food security. Results: Only 19.2\% of the households were comprised entirely of older adults. $63 \%$ of the examined households presented female headships. Food insecurity was $55 \%$, with higher rates in rural areas, lower socioeconomic strata, and households with more people ( $p$ $<0.05$ ); food insecurity was lower in households composed entirely of older adults $(\mathrm{OR}=0.58 ; \mathrm{p}<0.001)$, households with heads of household aged 60 years or older $(\mathrm{OR}=0.64 ; \mathrm{p}$ $=0.003$ ), and households without food aid programs for older adults $(\mathrm{OR}=0.33 ; \mathrm{p}<0.001)$. Conclusion: This study suggests a significant prevalence of food insecurity in households headed by older adults; furthermore, the head of household's age, gender, and whether the home is exclusively composed of older adults are all determinants of food insecurity.

-Keywords: elderly, sociodemographic factors, food insecurity, nutrition, public health, Medellín (Colombia).

\section{Resumo}

Objetivo: Determinar a insegurança alimentar e algumas características sociodemográficas associadas a ela, em lares de Medellin (Colômbia) nos quais habitam adultos com idades a partir de 60 anos. Metodologia: Estudo transversal, de amostragem aleatória estratificado, por nível socioeconômico e localização geográfica (urbano-rural). Este estudo foi baseado em 935 lares, nos quais habitava pelo menos um idoso. A segurança alimentar foi medida com a Escala Latinoamericana e Caribenha de Segurança Alimentar (ELCSA). Foram utilizadas o teste qui-quadrado $\left(\mathrm{X}^{2}\right)$ e odds ratio (OR), com intervalos de confiança para determinar a associação da segurança alimentar com as outras variáveis; além disso, foi elaborada uma árvore de classificação com o propósito de determinar a importância relativa das variáveis para explicar a segurança alimentar. Resultados: Um total de 19,2\% dos lares estava formado somente por idosos. $63 \%$ dos lares avaliados apresentavam chefes de famílias do sexo feminino. A insegurança alimentar foi de $55 \%$ com maiores proporções em zonas rurais, de baixa renda e quando se apresentou um maior número de pessoas por lar $(\mathrm{p}<0,05)$; a insegurança alimentar foi mais baixa em lares integrados somente por idosos (OR $=0,58 ; \mathrm{p}<0,001)$, com chefes de família de 60 anos ou mais $(\mathrm{OR}=0,64 ; \mathrm{p}=0,003)$ e lares que não contavam com programas de ajuda alimentar dirigidos a idosos (OR $=0,33 ; \mathrm{p}<0,001)$. Conclusões: Este estudo evidencia uma alta frequência de insegurança alimentar em lares onde vivem idosos; além da idade, o sexo do chefe de família e o fato de que esteja formado somente por idosos são aspectos determinantes da insegurança alimentar.

---------Palavras-chave: Idosos; Fatores sociodemográficos; Insegurança alimentar; Nutrição; Saúde pública; Medellin (Colômbia). 


\section{Introducción}

El proceso de envejecimiento presenta ritmos de crecimiento diferentes en países desarrollados y en vías de desarrollo. A pesar de esto, la tendencia en cualquier país es que los adultos mayores (AM) sean el grupo etario de mayor y más rápido aumento. En Colombia, para el año 2015, según datos de la "Encuesta de salud, bienestar y envejecimiento" (SABE), las personas de 60 años o más representaban el $11 \%$ de la población [1] y se espera, para el año 2025 , que esta proporción sea del $17,9 \%$ en el municipio de Medellín [2].

Este crecimiento poblacional traerá asociado un aumento de enfermedades crónicas, limitaciones y discapacidades, lo que impactará el acceso a los alimentos, tanto de las familias como de los AM, lo que a su vez podría derivar en inseguridad alimentaria (ISA) [3]. Este último concepto hace referencia a aspectos de alimentación y nutrición insuficientes, o una capacidad limitada de adquirir alimentos adecuados en formas socialmente aceptables. También es entendido como una inadecuada calidad o cantidad de alimentos en el hogar [4-6].

La ISA tiene una variedad de consecuencias para la salud, como la relación con las enfermedades crónicas y un pobre control metabólico, deterioro en la capacidad cognitiva y en la salud mental, además de alimentación menos saludable y escaso consumo de frutas, verduras y carnes magras [7-11]. Los efectos adversos son más severos en los AM, debido a que, ante la presencia de ISA en un hogar, se asumen mecanismos de subsistencia, como ingerir siempre un mismo alimento, consumir porciones más pequeñas o saltarse comidas, entre otras situaciones, lo que lleva a los integrantes del hogar a vivenciar el hambre [12].

La importancia que cobra la ISA en los AM se enmarca en la asociación que tiene con problemas económicos y con desprotección social, mostrando cómo la falta de dinero o de oportunidades para generar ingresos, crean condiciones desfavorables y mayor vulnerabilidad en la vejez.

Las publicaciones relacionadas con la ISA en hogares con AM en Colombia o en algunas de sus regiones, son escasas; sin embargo, diferentes estadísticas muestran la vulnerabilidad económica y social de la población AM y de sus hogares. Así, solo entre el 23 y el $31 \%$ cuentan con pensión [13]; además, el $30 \%$ de la población colombiana de 60 años o más, y el $7 \%$ de los mayores de 80, aún hacen parte del mercado laboral. Así mismo, para el año 2013, el 77,2 \% de las personas de 60 años o más no recibían ingresos, incluyendo pensiones, y un $3,6 \%$ recibió menos de un salario mínimo legal vigente [14], condiciones que impactan de manera adversa el estado nutricional.

De este modo, el objetivo de este estudio fue determinar la ISA y algunas características sociodemográficas asociadas a ella, en hogares de Medellín (Colombia) en los que habitan adultos de 60 años o más.

\section{Metodología}

Se realizó un estudio observacional transversal, en el marco del proyecto "Propuesta técnica para el desarrollo del perfil de seguridad alimentaria y nutricional 2015 y la asistencia técnica para la formulación del plan municipal de seguridad alimentaria y nutricional 2016-2028" [15].

La población estuvo conformada por los hogares de Medellín y sus corregimientos. La muestra para el estudio marco fue de 3040 hogares y se calculó mediante un muestreo aleatorio estratificado por condición socioeconómica y ubicación (urbana o rural) de la vivienda. Para dicho cálculo, se tuvo como base un error máximo permisible del $3 \%$, un nivel de confiabilidad del $95 \%$ y una prevalencia de seguridad alimentaria (SA) de 0,58. Se realizó un ajuste por no respuesta y se aplicó un efecto del diseño de 2.

De esta muestra evaluada, 935 hogares tenían al menos una persona AM viviendo de forma permanente en dicho hogar, quienes, según la Política Colombiana de Envejecimiento Humano y Vejez 2015-2024, son aquellas personas con 60 años o más [16].

Los datos de los individuos y de sus hogares fueron obtenidos por personal del área de la salud, de últimos semestres de formación o profesionales, capacitados en el ámbito de la seguridad alimentaria y utilizando técnicas estandarizadas.

\section{Variables}

En el presente estudio, la seguridad alimentaria y nutricional del hogar se consideró como variable dependiente y las características sociodemográficas como variables independientes.

La SA se evaluó por medio de la Escala Latinoamericana y Caribeña de Seguridad Alimentaria (ELCSA) [4]. Esta escala mide el acceso a los alimentos y determina el grado de SA basado en la experiencia en los hogares frente a esta condición.

La escala consta de 15 preguntas, relacionadas con la preocupación y la disponibilidad de dinero para compra de alimentos, la disminución de alimentos debido a la falta de dinero y la experiencia de hambre por parte de alguno de los integrantes del hogar. Cada una de las 15 preguntas es dicotómica: se da un puntaje de 1 a las respuestas afirmativas y 0 a las negativas.

De las preguntas, 8 aplican para hogares solo con adultos y la escala completa se aplica a los hogares con integrantes menores de 18 años. De acuerdo con estos códigos de respuesta, en hogares conformados solo por adultos, aquellos con puntaje de 0 se clasificaron como seguros; mientras que la inseguridad se puntuó así: de 
1 a 3, leve; de 4 a 6, moderada, y de 7 a 8 , severa. En hogares con integrantes menores de 18 años, aquellos con puntaje de 0 se clasificaron como seguros; 1 a 5 , inseguridad leve; 6 a 10, inseguridad moderada, y 11 a 15, inseguridad severa.

Factores sociodemográficos. Las características sociodemográficas incluyeron estrato socioeconómico (se refiere a la valoración que se realiza en Colombia y que va entre 1 y 6 de acuerdo con las características y condiciones de vida del barrio donde se encuentra ubicado el hogar, siendo 1 el más bajo y 6 el más alto); localización geográfica (urbana, rural); número de integrantes; ingreso mensual del hogar; sexo y edad del jefe del hogar; hacinamiento; número de adultos de 60 años y más que habitan el hogar; asistencia a programas de ayuda alimentaria dirigida a AM y hogar integrado únicamente por AM.

Estos datos, así como los relacionados con la SA, fueron suministrados por el jefe del hogar o una persona mayor de 18 años, que tuviera conocimiento de la adquisición de alimentos del hogar.

\section{Análisis estadístico}

Los análisis fueron desarrollados en el software sPss versión 24 (Chicago: SPSS Inc.; 2016), licencia de la Escuela de Nutrición y Dietética de la Universidad de Antioquia.

Las variables se describieron mediante frecuencias absolutas y relativas.

Las características de SA se estratificaron por los factores sociodemográficos y su asociación se determinó por medio de la prueba de chi cuadrado $\left(\chi^{2}\right)$ de independencia y los odds ratio con sus respectivos intervalos de confianza (IC) del $95 \%$.

Un árbol de clasificación fue realizado para analizar el comportamiento de las categorías de SA con relación a los diferentes factores sociodemográficos, así como la interacción entre estos últimos. Valores de $p<0,05$ fueron asumidos como significativos.

\section{Consideraciones éticas}

Según los principios de la declaración de Helsinki [17] y el Ministerio de Salud de Colombia en la Resolución 008430 de octubre de 1993, artículo 11 [18], la investigación se clasificó de riesgo mínimo, ya que la toma de los datos no implicó riesgos para la integridad física o mental de los participantes.

Este proyecto fue aprobado por el Comité de Bioética de la Facultad de Odontología de la Universidad de Antioquia, según Acta 01 del 27 de febrero de 2015.

El jefe del hogar o la persona mayor de 18 años que contestó la encuesta firmó el consentimiento informado, aceptando la participación voluntaria en el estudio.

\section{Resultados}

Del total de hogares evaluados $(n=935)$, el $88,1 \%$ pertenecía al área urbana, en su mayoría de estratos socioeconómicos 1 y $2(62,6 \%)$, conformados por 2 a 4 integrantes $(57,6 \%)$ y con jefaturas femeninas $(63,0 \%)$ (véase Tabla 1).

Tabla 1. Características generales de los hogares con adultos mayores según clasificación de seguridad alimentaria

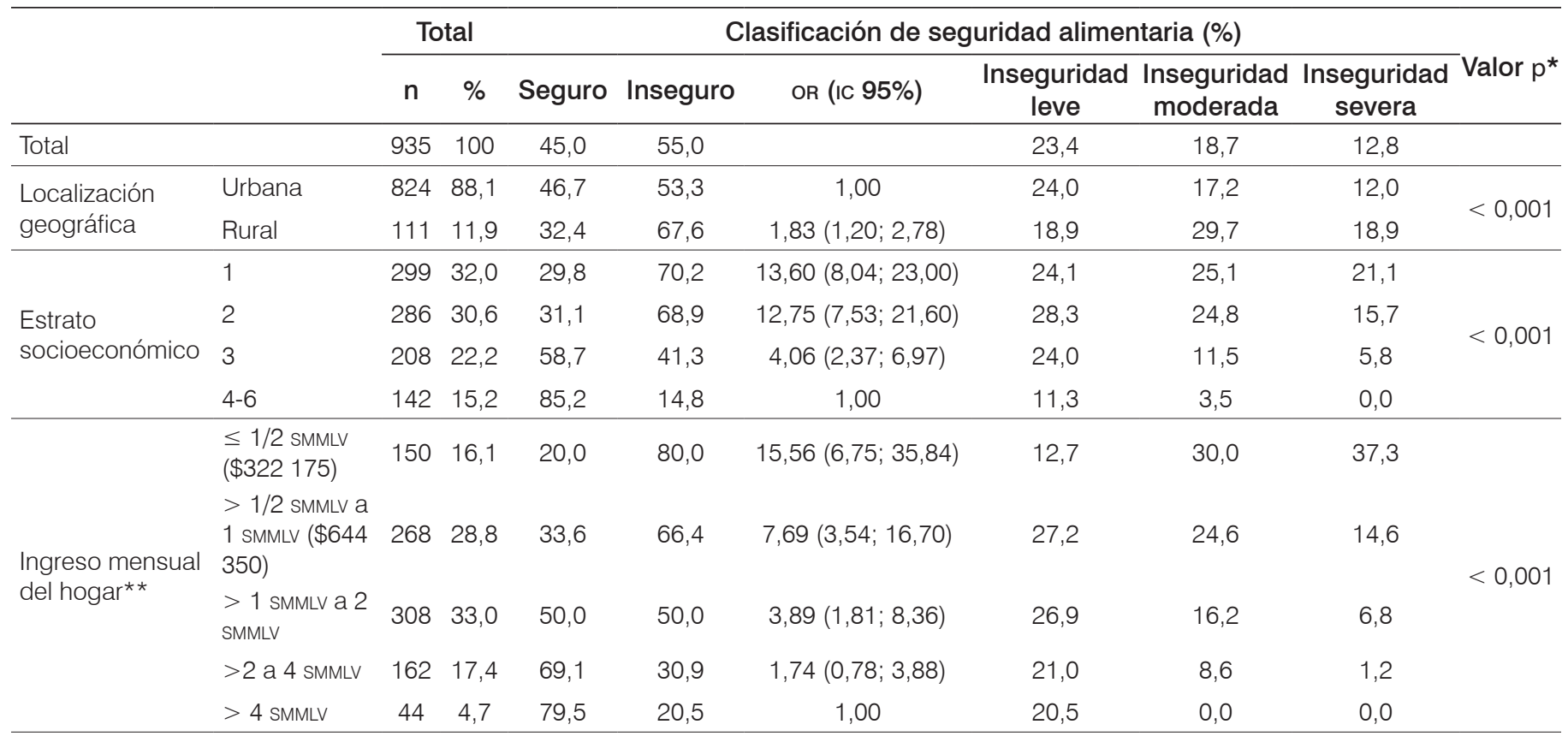




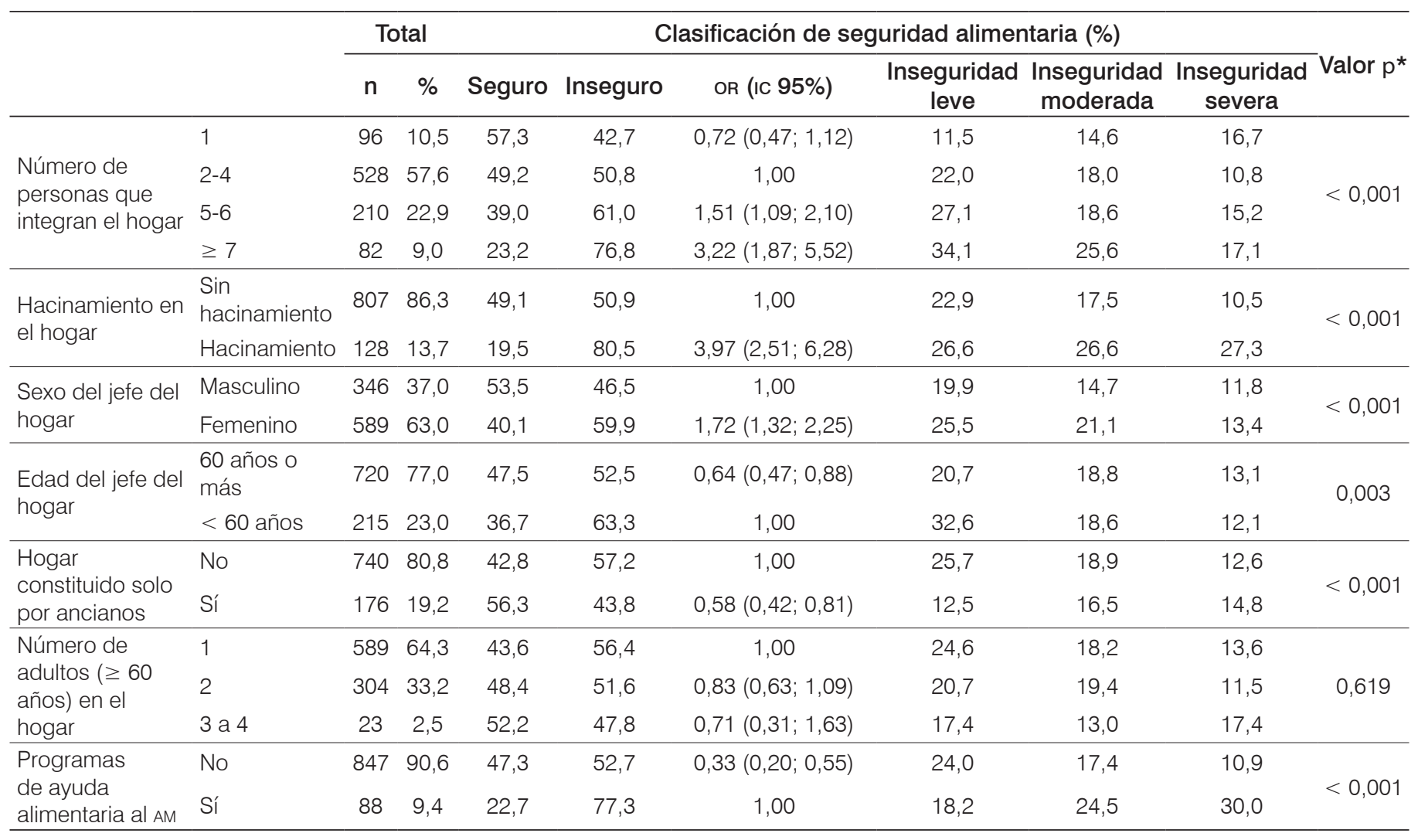

AM: Adulto mayor; IC: intervalo de confianza; oR: odds ratio; SMMLv: salario mínimo mensual legal vigente

* Prueba chi cuadrado de independencia.

** El valor del SMMLV para Colombia en 2015 era de $\$ 644350$ cop.

En los hogares, se encontró que el 64,3\% tenía un AM como integrante del mismo; el 33,2\% tenía dos AM y solo el $2,5 \%$ contaba con tres o más. De igual manera, el $19,2 \%$ estaba constituido exclusivamente por AM. El 9,4 $\%$ de los hogares se hallaba inscrito en algún programa de ayuda alimentaria dirigido a este grupo.

La ISA en los hogares de AM en Medellín fue del 55,0 $\%$. Este porcentaje fue más alto en la zona rural $(67,6 \%$, $p<0,001)$. Además, se halló una relación inversa entre ISA y estrato socioeconómico $(p<0,01)$, pero una asociación positiva con número de personas que integran el hogar $(p<0,001)$.

Otras características asociadas con menores porcentajes de ISA fueron: hogares integrados solo por AM (OR $=0,58$; IC $95 \%$ : 0,42-0,81), jefes del hogar con edades de 60 años o más (OR $=0,64$; IC $95 \%$ : 0,47-0,88) y no contar con algún programa de ayuda alimentaria dirigido a $\mathrm{AM}(\mathrm{OR}=0,33$; IC $95 \%: 0,20-0,55)$ (véase Tabla 1).

La ISA severa fue del $12,8 \%$, situación más pronunciada en áreas rurales, estratos bajos, hogares con ha- cinamiento y aquellos que contaban con programas de ayuda alimentaria para dicho grupo.

Después de realizar un árbol de clasificación (véase Figura 1), se observó que la ISA aumentó 6 puntos porcentuales (pasó de 55,0 a 61,3\%) cuando la edad del jefe del hogar era de 60 años o más, el hogar era de estratos 1 y 2, no tenía hacinamiento, jefatura del hogar femenina y estaba constituido solo por AM; pero aumentó 18 puntos porcentuales (de 55,0 a 72,7 \%) en hogares que no estaban constituidos solo por AM.

De igual manera, cuando el jefe del hogar tenía una edad de 60 años o más, vivía en estrato 3 y era del sexo masculino, la ISA disminuyó 24 puntos porcentuales (de 55,0 pasó a 30,5 \%); mientras que cuando el jefe del hogar era del sexo femenino, la disminución fue de 10 puntos (de 55,0 a 45,0 \%).

Finalmente, hogares con jefes de menos de 60 años y de estratos 1 y 2 , aumentaron la ISA en 18 puntos porcentuales (de 55,0\% pasaron a 73,0 \%). 


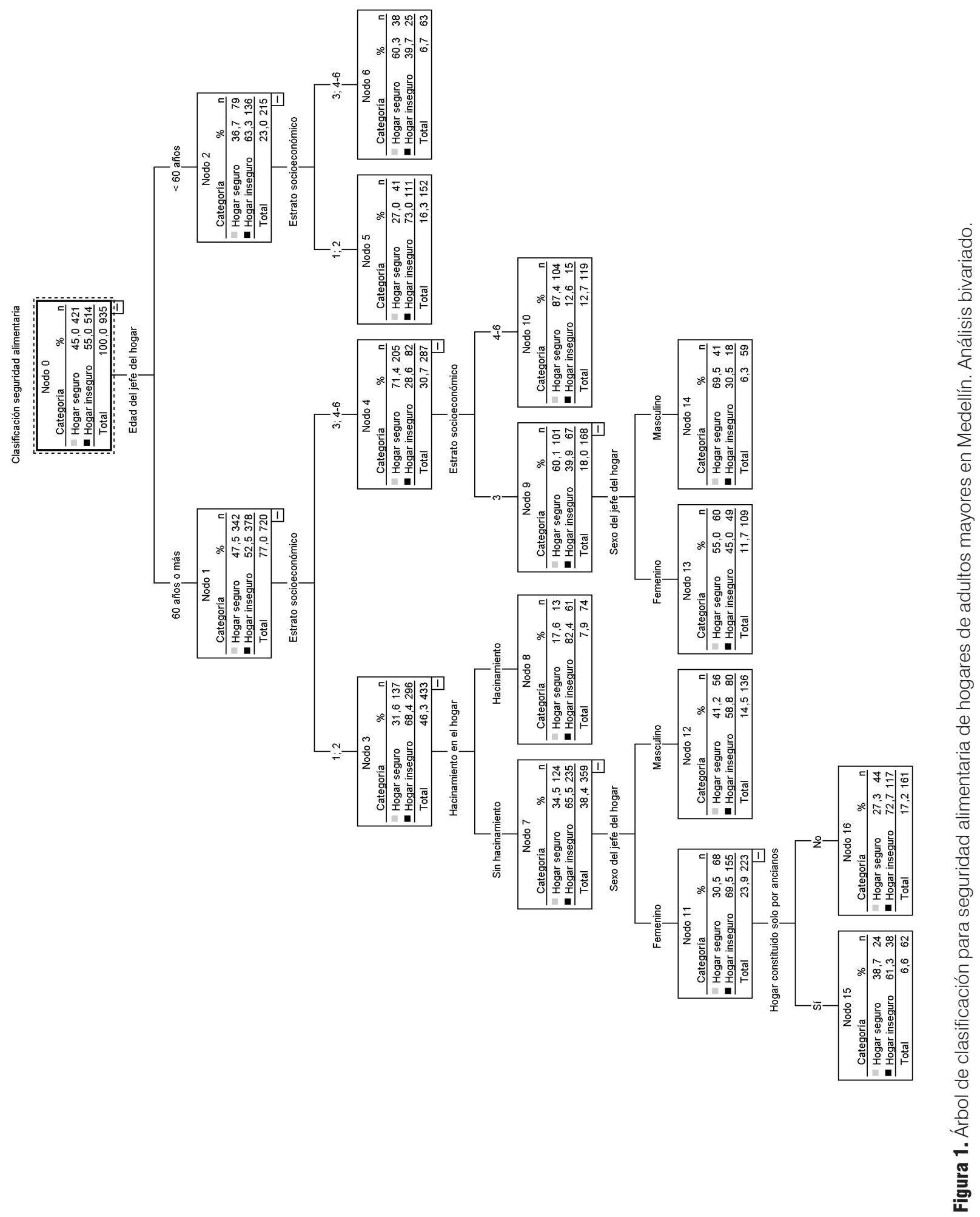

6 | Universidad de Antioquia 


\section{Discusión}

La frecuencia de ISA en este estudio fue similar a la hallada en Colombia, tanto para el año $2010(57,7 \%)$ como para el 2015 (54,2 \%) [19]; sin embargo, el grado de severidad de la ISA hallada en Medellín fue mayor en comparación con los hogares colombianos. La edad y el sexo del jefe, así como hogares conformados solo por $\mathrm{AM}$, fueron aspectos determinantes para la ISA.

Este estudio mostró resultados similares a los hogares de Georgia (Estados Unidos), en su gran mayoría con AM, y en hogares de Brasil con AM. En Georgia, el $52,1 \%$ se clasificó en ISA; además, el vivir en estratos bajos, participar en programas de alimentación para el AM y jefatura de hogar femenina, se asociaron a mayores porcentajes de dicha condición [20]. En el estudio de Brasil, la frecuencia de ISA fue también del 52,0 \%, siendo mayor en estratos bajos; además, estos hogares presentaron menores promedios de ingresos monetarios que los hogares seguros [21].

En Chile, Vargas et al. encontraron que el 40,4 \% de los hogares manifestaron algún grado de ISA; a pesar de ser un porcentaje menor al hallado en Medellín, aspectos como el sexo femenino, familias más numerosas, menores ingresos y pertenecer al estrato medio-bajo o bajo, también evidenciaron mayores porcentajes de ISA [12]. En esta misma línea, en Portugal, un estudio encontró una baja prevalencia de ISA (16,5\%); sin embargo, las mujeres y los hogares con bajos ingresos mostraron mayores OR para esta, hallazgos similares a los reportados en este estudio [22]; mientras que, en México, se reportó que la frecuencia de IsA fue mayor a la de Medellín, con cifras de $68,6 \%$ en los hogares con AM, posiblemente porque los participantes eran beneficiarios de ayudas alimentarias [23].

La jefatura de hogar femenina se asoció con mayores frecuencias de ISA en hogares en general. Esta condición viene aumentando en Colombia, pues en el 2003 era del $28,6 \%$; en 2014, del 34,7\% [24], y en 2018, del $36,8 \%$ [25]. En los hogares con AM, el porcentaje de jefatura femenina pasó de 35,5\% en el 2003 a 42,2 \% en 2014 [26].

Es importante resaltar que las mujeres presentan mayor frecuencia de pobreza en la población en general y entre AM, lo que se ha denominado una "feminización de la pobreza", situación que conduce a que las mujeres presenten mayores dificultades para acceder a una alimentación de mejor calidad nutricional [27].

La ISA contribuye a riesgo nutricional en la población AM; afecta de forma negativa el bienestar psicológico y social, e incrementa la demanda de servicios de salud, lo que genera consecuencias económicas considerables $[5,28,29]$.
En Estados Unidos, por ejemplo, Nord [30] reportó que la prevalencia de ISA entre los AM es baja, gracias al establecimiento de ingresos suministrados por programas de seguridad social, pensiones y los programas de asistencia de alimentos comunitarios y nacionales. Así mismo, Ratcliffe et al. [31] reportaron que participar en el programa de asistencia alimentaria estuvo asociado con menos ISA. Contrario a esto, otros investigadores, como Goldberg y Mawn [32] y Lee y Frongillo [33], mostraron que los hogares o participantes que recibieron beneficios del programa de asistencia alimentaria fueron más propensos a ISA, comparados con aquellos que nunca habían recibido este beneficio. Los resultados de estas dos últimas investigaciones están acordes con lo hallado en nuestro estudio.

Algunas situaciones que ayudan a entender la alta frecuencia de ISA en AM que reciben los programas de alimentación son las caídas repentinas en los ingresos económicos del hogar, falta de vinculación al sistema de pensiones, además que los recursos suministrados por los programas pueden ser insuficientes. En Colombia, solo alrededor de una cuarta parte de la población AM recibe una pensión [13]. Además, se estima que el 48,7 $\%$ de las personas ocupadas tienen un trabajo informal, lo que implica una menor protección social y una disminución en el bienestar de las poblaciones [14].

Otro aspecto importante de resaltar es que la ayuda alimentaria o monetaria de los programas del Estado se destina para satisfacer otras necesidades o se diluye en los demás integrantes del hogar y no alcanza a reducir la ISA, entre otros factores, por el número de integrantes, por los ingresos económicos escasos o porque los subsidios económicos del Gobierno, si bien son un aporte importante, no logran cubrir las necesidades alimentarias de los hogares, pues, por ejemplo, para el año 2015, según el Manual operativo - Anexo Técnico No. 2 del Ministerio del Trabajo [34], el aporte económico mensual que hacía el programa "Colombia Mayor" era de $\$ 75000$ cop (us\$27,34), lo que representaba el 11,6\% del salario mínimo mensual en Colombia.

Un punto que se resalta en este estudio es que la ISA fue menor en hogares con jefaturas de personas de 60 años o más de edad. Esto podría deberse a que los ingresos percibidos por los AM se convierten en una contribución significativa, que ayuda a proteger el hogar de la ISA; además, se ha documentado que cuando el $\mathrm{Am}$ es el jefe del hogar, hay un uso más racional de los recursos [26].

Por otro lado, este estudio, así como otros realizados en hogares con AM [32,35-37] y en hogares sin ellos [12,38-39], lograron evidenciar una asociación entre zona rural, bajos estratos socioeconómicos y mayores porcentajes de ISA, a pesar de que algunos utilizaron instrumentos de medida de la sA diferentes al nuestro. Dentro de estos instrumentos se pueden mencionar el módulo de inseguridad alimentaria en el hogar de Esta- 
dos Unidos (Household Food Security Survey Module), la escala brasilera de inseguridad alimentaria (EBIA), la escala de inseguridad en el acceso a los alimentos en el hogar (Household Food Insecurity Access Scale HFIAS-), entre otros [40].

Dentro de las limitaciones de esta investigación se encuentran el diseño trasversal, que no permite inferir causalidad; la muestra de la población AM del proyecto marco, que no fue representativa de la ciudad, y la ISA, QUE se enfocó solo en la falta de recursos económicos, pues en otros estudios [21,41] se han considerado otras causas, como la incapacidad para comprar alimentos o cocinar, lo que puede llevar a subreporte de ISA en esta población.

\section{Conclusión}

Este estudio mostró una frecuencia importante de ISA en hogares donde habitan de forma permanente AM; los hogares con jefes menores de 60 años, con jefaturas femeninas, mostraron mayores frecuencias de ISA, mientras que aquellos integrados solo por AM redujeron la posibilidad de presentar ISA.

El creciente número de personas de 60 años y más, la alta frecuencia de ISA en los hogares con AM y los factores asociados a esta muestran la importancia de considerarla un desafío para la salud pública y una oportunidad para la realimentación de las políticas públicas para la ciudad. Se debe fortalecer el trabajo entre los entes gubernamentales, la academia y la sociedad civil, con miras a establecer mecanismos que empoderen a este grupo poblacional y a sus familias, con el fin de mejorar el acceso a los alimentos, escenario que se torna cada vez más complejo, no solo en lo económico, sino también en lo social, desde la integración y el reconocimiento de sus saberes y habilidades.

\section{Agradecimientos}

Los investigadores agradecen a la Alcaldía de Medellín, a su Secretaría de Inclusión Social, Familia y Derechos Humanos, y al Equipo de Seguridad Alimentaria de dicha Secretaría, por permitir el acceso a la base de datos para la realización de este estudio. También agradecen al Centro de investigación en Alimentación y Nutrición de la Escuela de Nutrición y Dietética de la Universidad de Antioquia, por su apoyo logístico.

\section{Financiación}

Esta investigación fue financiada por la Alcaldía de Medellín, a través de la Secretaría de Inclusión Social, Familia y Derechos Humanos. Número de contrato interadministrativo 4600058538 de 2015.

\section{Conflicto de intereses}

Los autores manifiestan que no hubo conflicto de interés entre la población evaluada, la entidad financiadora y el grupo investigador.

\section{Declaración de responsabilidad}

Los autores declaran que los puntos de vista expresados son de su total responsabilidad y no de la institución en la que trabajan.

\section{Declaración de autoría}

Todos los autores participaron en la depuración de la base de datos, la interpretación, el análisis de los datos y la discusión de los resultados, así como en la escritura del artículo. Los autores se hacen responsables del manuscrito presentado para publicación.

\section{Referencias}

1. Colombia, Ministerio de Salud y Protección Social (Minsalud), Departamento Administrativo de Ciencia Tecnología e Innovación, Colciencias, Universidad del Valle, Universidad de Caldas. Encuesta de salud, bienestar y envejecimiento (SABE) Bogotá: Minsalud; 2016.

2. Departamento Administrativo Nacional de Estadísticas. Proyecciones de población municipal por área, sexo y edad, para el periodo 2018-2026 [internet]. 2020 [consultado 2021 jun. 20]. Disponible en: https://www.dane.gov.co/index.php/estadisticas-por-tema/ demografia-y-poblacion/proyecciones-de-poblacion

3. Vilar-Compte M, Gaitán-Rossi P, Pérez-Escamilla R. Food insecurity measurement among older adults: Implications for policy and food security governance. Global Food Security. 2017;14:8795. DoI: https://doi.org/10.1016/j.gfs.2017.05.003

4. Segall AM, Álvarez MC, et al. Escala Latinoamericana y Caribeña de Seguridad Alimentaria(ELCSA). Manual de uso y aplicación. Santiago de Chile: Organización de las Naciones Unidas para la Alimentación y la Agricultura (FAO); 2012.

5. Alaimo K, Chilton M, Jones SJ. Food insecurity, hunger and malnutrition. En: Marriot B, Birt DF, Stallings VA, Yates AA. Present knowledge in nutrition. Clinical and applied topics in nutrition. Volume 2. 11. ${ }^{\mathrm{a}}$ ed. London: Elsevier; 2020. pp. 311-26.

6. Franklin B, Jones A, Love D, et al. Exploring mediators of food insecurity and obesity: A review of recent literature. J Community Health. 2012;37(1):253-64. Dor: https://doi.org/10.1007/s10900-011-9420-4

7. Guerrero N, Walsh MC, et al. Urban-rural and regional variability in the prevalence of food insecurity: The survey of the health of Wisconsin. Wis Med J. 2014;113(4):133-8.

8. Ramsey R, Giskes K, et al. Food insecurity among adults residing in disadvantaged urban areas: Potential health and dietary consequences. Public Health Nutr. 2012;15(02):227-37. DoI: https:// doi.org/10.1017/S1368980011001996

9. Fernandes SG, Rodrigues AM, Nunes C, et al. Food insecurity in older adults: Results from the epidemiology of chronic diseases cohort study 3. Front. Med. 2018; 5:203. Dor: https://doi. org/10.3389/fmed.2018.00203 
10. Leung CW, Epel ES, Ritchie LD, et al. Food insecurity is inversely associated with diet quality of lower-income adults. J Acad Nutr Diet. 2014;114(12):1943-53.e2. Dor: https://doi.org/10.1016/j. jand.2014.06.353

11. Frith E, Loprinzi PD. Food insecurity and cognitive function in older adults: Brief report. Clin Nutr. 2018;37(5):1765-8. DoI: https://doi.org/10.1016/j.clnu.2017.07.001

12. Vargas Puello V, Alvarado Orellana S, Atalah Samur E. Inseguridad alimentaria en adultos mayores en 15 comunas del gran Santiago; un tema pendiente. Nutr Hosp. 2013;28(5):1430-7. DoI: https://dx.doi.org/10.3305/nh.2013.28.5.6663

13. Villar L, Flórez CE, Forero D, et al. Protección económica a la población mayor en Colombia. En: Fundación para la Educación Superior y el Desarrollo (Fedesarrollo) y Fundación Saldarriaga Concha, Misión Colombia envejece: cifras, retos y recomendaciones. Bogotá: Editorial Fundación Saldarriaga Concha [internet]; 2015 [citado 2020 oct. 5]. pp. 84-165. Disponible en: https:// www.fedesarrollo.org.co/sites/default/files/envejece.pdf

14. Martínez-Restrepo S, Enríquez E, Pertuz MC AJ. El mercado laboral y las personas mayores. En: Fundación para la Educación Superior y el Desarrollo (Fedesarrollo) y Fundación Saldarriaga Concha, Misión Colombia envejece: cifras, retos y recomendaciones. Bogotá: Editorial Fundación Saldarriaga Concha [internet]; 2015 [citado 2020 oct. 5]. pp. 166-243. Disponible en: https:// www.fedesarrollo.org.co/sites/default/files/envejece.pdf

15. Hoyos GM, Álvarez MC, et al. Metodología. En: Alcaldía de Medellín y Universidad de Antioquia (Ed.), Perfil de seguridad alimentaria y nutricional de Medellín y sus corregimientos 2015 . Medellín - Colombia; 2015. pp. 11-51.

16. Colombia, Ministerio de Salud. Política colombiana de envejecimiento humano y vejez 2015-2024 [internet]. 2015 [citado 2019 jul. 25]. Disponible en: https://www.minsalud.gov.co/sites/rid/ Lists/BibliotecaDigital/RIDE/DE/PS/Pol\%C3\%ADtica-colombiana-envejecimiento-humano-vejez-2015-2024.pdf

17. World Medical Association. World Medical Association Declaration of Helsinki: Ethical principles for medical research involving human subjects. JAMA. 2013; 310(20):2191-4. DOI: https://doi. org/10.1001/jama.2013.281053

18. Colombia, Ministerio de Salud. Resolución 8430, por la cual se establecen las normas científicas, técnicas y administrativas para la investigación en salud (1993 oct. 4).

19. Universidad Nacional de Colombia, Instituto Nacional de Salud, Instituto Colombiano de Bienestar Familiar, Ministerio de Salud y Protección Social. Encuesta nacional de la situación nutricional en Colombia (ENSIN) 2015. Bogotá: Instituto Colombiano de Bienestar Familiar; 2019.

20. Lee JS, Johnson MA, et al. Food security of older adults requesting Older Americans Act Nutrition Program in Georgia can be validly measured using a short form of the U.S. Household Food Security Survey Module. J Nutr. 2011;141(7):1362-8. Dor: https:// doi.org/10.3945/jn.111.139378

21. Marín-León L, Segal-Corrêa AM, Panigassi G, et al. A percepção de insegurança alimentar em famílias com idosos em Campinas, São Paulo, Brasil. Cad Saude Publica. 2005; 21(5):1433-40. DoI: https://doi.org/10.1590/s0102-311x2005000500016

22. Maia I,Monjardino T,Frias B, etal.Food insecurity in Portugal among middle- and older-aged adults at a time of economic crisis recovery: Prevalence and determinants. Food Nutr Bull. 2019;40(4):504-13. DOI: https://doi.org/10.1177/0379572119858170

23. Rivera-Márquez JA, Mundo-Rosas V, et al. Inseguridad alimentaria en el hogar y estado de nutrición en personas adultas mayores de México. Salud Publica Méx. 2014;56(Supl. 1):71-8.
24. Departamento Nacional de Planeación, Observatorio de Políticas de las Familias. Tipologías de familias en Colombia: evolución 1993-2014. Documento de trabajo No. 2016-1. Bogotá [internet]; 2015 [citado 2020 oct. 5]. Disponible en: https://observatoriodefamilia.dnp.gov.co/Documents/Documentos de trabajo/D3-tipologias-evolucion_dic3-(1).pdf

25. Departamento Administrativo Nacional de Estadística. Serie de indicadores Encuesta de calidad de vida 2003-2018 [internet]; 2018 [citado 2020 oct. 5]. Disponible en: https://www.dane.gov. co/index.php/estadisticas-por-tema/salud/calidad-de-vida-ecv/ encuesta-nacional-de-calidad-de-vida-ecv-2018

26. Departamento Nacional de Planeación. Los adultos mayores en el hogar como proveedores y demandantes de cuidado. En Observatorio de políticas de las familias. Boletín 6, diciembre de 2015. Bogotá [internet]; 2015 [citado 2020 oct. 5]. pp. 38-45. Disponible en: https://observatoriodefamilia.dnp.gov.co/Documents/ Boletines/bolet\%C3\%ADn-no-6_vf.pdf

27. FAO; OPS; WFP; UNICEF. Panorama de la seguridad alimentaria y nutricional en América Latina y el Caribe 2018. Santiago de Chile [internet]; 2018 [citado 2020 feb. 22]. Disponible en: http:// www.fao.org/3/CA2127ES/ca2127es.pdf

28. Steiner JF, Stenmark SH, Sterrett AT, et al. Food insecurity in older adults in an integrated health care system. J Am Geriatr Soc. 2018;66(5):1017-24. DoI: https://doi.org/10.1111/jgs.15285

29. Pooler JA, Hartline-Grafton H, DeBor M, et al. Food insecurity: A key social determinant of health for older adults. J Am Geriatr Soc. 2019;67(3):421-4. DoI: https://doi.org/10.1111/jgs.15736

30. Nord M. Food security rates are high for elderly households. Food Rev. 2002;25(2):19-24. DoI: https://doi.org/10.22004/ ag.econ. 234603

31. Ratcliffe C, McKernan M, Zhang S. How much does the supplemental nutrition assistance program reduce food insecurity? Am J Agric Econ. 2011;93(4):1082-98. DoI: https://doi.org/10.1093/ajae/aar026

32. Goldberg SL, Mawn BE. Predictors of food insecurity among older adults in the United States. Public Health Nurs. 2015;32(5):397407. DoI: https://doi.org/10.1111/phn.12173

33. Lee JS, Frongillo EA. Factors associated with food insecurity among U.S. elderly persons: Importance of functional impairments. J Gerontol B Psychol Sci Soc Sci. 2001;56(2):S94-9. DOI: https://doi.org/10.1093/geronb/56.2.s94

34. Colombia, Ministerio del Trabajo. Programa de protección social al adulto mayor - Hoy Colombia Mayor-. Manual operativo, anexo técnico 2. Bogotá; [internet] 2015 [citado 2020 oct. 5]. Disponible en: https://www.fondodesolidaridadpensional.gov.co/manualoperativo/74-anexo-tecnico-no2-manual-operativo/file.html.

35. Dean WR, Sharkey JR, Johnson CM. Food insecurity is associated with social capital, perceived personal disparity, and partnership status among older and senior adults in a largely rural area of central Texas. J Nutr Gerontol Geriatr. 2011;30(2):169-86. DoI: https://doi.org/10.1080/21551197.2011.567955

36. Souza BF de, Marín-León L, et al. Demographic and socioeconomic conditions associated with food insecurity in households in Campinas, SP, Brazil. Rev Nutr. 2016;29(6):845-57. DoI: https:// doi.org/10.1590/1678-98652016000600009

37. Chinnakali P, Upadhyay RP, Shokeen D, et al. Prevalence of household-level food insecurity and its determinants in an urban resettlement colony in north India. J Health Popul Nutr. 2014;32(2):227-36.

38. Gholami A, Sani TR, Askari M, et al. Food insecurity status and associated factors among rural households in north-east of Iran. Int J Prev Med. 2013;4(9):1018-24. 
39. Birhane T, Shiferaw S, Hagos S, Mohindra KS. Urban food insecurity in the context of high food prices: A community based cross sectional study in Addis Ababa, Ethiopia. BMC Public Health. 2014;14:680. DOI: https://doi.org/10.1186/1471-2458-14-680

40. Marques, E., Reichenheim, M., De Moraes, C., Antunes, M., \& Salles-Costa, R. (2015). Household food insecurity: A systematic review of the measuring instruments used in epidemiological studies. Public Health Nutrition, 18(5), 877-892. Dor:10.1017/ S1368980014001050

41. Gajda R, Jeżewska-Zychowicz M. Elderly Perception of Distance to the Grocery Store as a Reason for Feeling Food InsecurityCan Food Policy Limit This? Nutrients. 2020; 12(10): 3191. DoI: 10.3390/nu12103191 